\title{
Ácaros asociados a dos especies de Odontotaenius (Coleoptera: Passalidae) en México
}

\section{Pilar Liliana Barrios-Torres \& Gabriel A. Villegas-Guzmán}

Laboratorio de Acarología "Dra. Isabel Bassols Batalla”. Escuela Nacional de Ciencias Biológicas, IPN. Prolongación del Carpio, esquina con Plan de Ayala s/n, Casco de Santo Tomás, México, D. F., C. P. 11340; plilianabtorres777@hotmail.com, gabrvill@yahoo.com

\author{
Recibido 04-IX-2014. C Corregido 14-II-2015. Aceptado 12-III-2015.
}

\begin{abstract}
Mites associated with two species of the genus Odontotaenius (Coleoptera: Passalidae) in Mexico. Mites can establish association with different arthropods as coleopterans families Scarabaeidae and Passalidae. Passalids are distributed in tropical and templates zones, and until now, more than 200 species of mites have been associated to them. One of the relationships between passalids and mites is the phoresy where one small animal (the phoretic) seeks out and attaches to another animal (the host) for transportation. Herein, we studied the mites associated to $O$. zodiacus and $O$. striatopunctatus; for this, 80 Odontotaenius with mites were reviewed; passalids were collected in and under decaying logs from six states of Mexico, and were individually kept in vials with $80 \%$ ethanol. The specimens were carried to the laboratory and mites removed with fine-pointed forceps under stereo microscope. The mites were stored with $80 \%$ alcohol until some were cleared with lacto-phenol and mounted in Hoyer's solution. We found 1945 mites belonging to 13 families (Acaridae, Ascidae, Diarthrophallidae, Digamasellidae, Diplogyniidae, Euzerconidae, Heterocheylidae, Histiostomatidae, Klinckowstroemiidae, Laelapidae, Megisthanidae, Trematuridae, and Uropodidae) and 42 species, being the

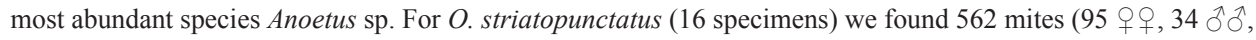
197 hypopus, 234 deutonymph, 2 tritonymph) of 11 families and 22 species; the most abundant were Uropodidae (42\%) and Histiostomatidae (26\%). While for O. zodiacus (64 specimens) were found 1383 mites (300 $\circ$, $204 \widehat{\partial}, 608$ hypopus, 139 deutonymphs, 133 tritonymphs) of 10 families and 30 species; the most abundant were: Diartrophallidae, Acaridae, and Histiostomatidae (23\% for the two first and $21 \%$ for third). The high abundance and richness was in O. zodiacus, likewise Margalef $\left(\mathbf{S}^{\prime}\right)$ and Shanon-Winner $\left(\mathbf{H}^{\prime}\right)$ indexes were higher in this species $\left(O\right.$. zodiacus $\mathbf{S}^{\prime}=4.05, \mathbf{H}^{\prime}=2.2 ; O$ striatopunctatus $\left.\mathbf{S}^{\prime}=3.34, \mathbf{H}^{\prime}=1.94\right)$, while Equity $\left(\mathrm{E}_{\mathrm{H}}\right)$ was similar to both hosts (O. zodiacus $\mathbf{E}_{\mathbf{H}}=0.64$; O. striatopunctatus $\mathbf{E}_{\mathbf{H}}=0.63$ ). The mites were found principally in the protected zone of the passalid's body, as under elytron, membranous wings, and in the clefts of the ventral region of the passalid, and, in minor proportion, on the head and coxal regions. From our findings, 12 species were new records for the Mexican passalids: Abrotarsala cuneiformis, A. obesa, A. pyriformis, Brachytremella sp., Diarthrophallus cartwrighti, D. crinatus, Lombardiniella sp., Trichodiplogynium carlosi, $T$. sahlbergi, T. hirsutum and Trichodiplogynium sp., and additional studies may help describe and understand these mites-passalids associations. Rev. Biol. Trop. 63 (3): 659-671. Epub 2015 September 01.
\end{abstract}

Key words: mites, diversity, passalids beetles, new records, distribution.

Los ácaros son un grupo abundante y diverso, es posible encontrarlos en vida libre o como simbiontes permanentes y temporales (Paracer \& Ahmadjian, 2000). Pueden establecer asociaciones de parasitismo, comensalismo y mutualismo con diferentes tipos de animales incluyendo mamíferos, aves e insectos; entre estos últimos encontramos a los coleópteros de la familia Passalidae.

Los pasálidos se encuentran distribuidos en las zonas tropicales y templado-húmedas del mundo (Reyes-Castillo, 2004; Amat-García, Blanco, \& Reyes Castillo, 2004). Existen 680 especies que conforman 61 géneros 
(Reyes-Castillo, Rojas-Gómez, \& Vázquez, 2006). Todos sus miembros tienen requerimientos ecológicos y alimenticios similares. Tienen hábitos subcortícolas, saproxilófagos y desarrollan todo su ciclo de vida dentro de los troncos en descomposición; los adultos salen sólo para dispersarse (Reyes-Castillo \& Halffter, 1984; Reyes-Castillo, 2004; ReyesCastillo et al., 2006). En México, hay registros de 104 especies en 22 géneros, se distribuyen en bosque mesófilo de montaña y ecosistemas forestales tropicales a altitudes que van de los 0 a los $3000 \mathrm{msnm}$, donde se encuentra la mayor cantidad de especies, su distribución disminuye en bosques tropicales caducifolios y subcaducifolios (Castillo \& Reyes-Castillo, 2003; Reyes-Castillo et al., 2006).

Odontotaenius se distribuyen en América, desde Canadá hasta Panamá. Cuenta con sólo diez especies, seis de ellas registradas para México (Reyes-Castillo \& Amat-García, 2003; Reyes-Castillo et al., 2006). Entre ellas se encuentran Odontotaenius striatopunctatus Percheron 1835 y O. zodiacus Truqui 1857.

$O$. striatopunctatus es una especie de amplia distribución geográfica, que ha sido registrada para 16 estados de la República Mexicana, se encuentra en ecosistemas como selva alta perennifolia, selva mediana subcaducifolia, selva baja caducifolia, bosque de encino y bosque mesófilo de montaña a un intervalo altitudinal entre 5 y $2400 \mathrm{msnm}$, desde el Noreste de México hasta Colombia. Puede llegar a medir en promedio de 23 a 27 $\mathrm{mm}$ de largo (Reyes-Castillo, 2000; ReyesCastillo \& Amat-García, 2003; Reyes-Castillo et al., 2006).

O. zodiacus es endémico de México y se distribuye en los estados de Hidalgo, Puebla y Veracruz, tanto en bosque mesófilo de montaña como en bosque de pino y encino a una altitud de 1000 a 2390 msnm. Es posible distinguirlo por una mayor longitud (30-38 mm) (Castillo, Rivera-Cervantes, \& Reyes-Castillo, 1988; Reyes-Castillo et al, 2006).

Se conocen más de 200 especies de ácaros asociadas con coleópteros pasálidos, abarcando 68 géneros y 21 familias de los órdenes
Mesostigmata, Trombidiformes y Sarcoptiformes (Hunter, 1993a). Una asociación importante entre ácaros y pasálidos es la foresia, que consiste en el transporte de un organismo pequeño (foronte) quien se fija temporalmente a un organismo más grande (huésped) con el fin de dispersarse, esto inducido por cambios en el microclima o a una alta densidad poblacional (Binns, 1981; Hunter, 1993a). En esta asociación, el huésped no resulta perjudicado, siempre y cuando la densidad del foronte no impida el movimiento del mismo; por otra parte, garantiza al foronte la colonización de nuevos hábitats (Athias-Binche, 1994). La foresia por el sitio de unión al huésped puede ser de dos tipos: activa, cuando el foronte se fija a zonas expuestas del huésped, y pasiva, cuando se aprovechan las cavidades del mismo (Szymkowiak, Górski, \& Bajerlein, 2007). La foresia ocurre en diferentes estadíos dependiendo de la familia, en estadíos inmaduros y adultos (principalmente las hembras). Algunas deutoninfas se han adaptado morfológicamente en los quelíceros, pedipalpos, sedas (Laelapidae), carúnculas (Euzerconidae, Megisthanidae y Diplogyniidae) o glándulas bucales (larvas de Hydracariens) que les permiten sujetarse al huésped; además del desarrollo de un pedicelo anal (Uropodidae) o ventosas adherentes (hipopodios de Acaridae), todo para llevar a cabo la fijación y el transporte (Athias-Binche, 1994).

Se ha registrado la relación de ácaros asociados a 22 especies de pasálidos en México (Trägärdh, 1950; Delfinado \& Baker, 1975; Schuster \& Summers, 1978; Hunter, 1993b; Villegas-Guzmán, Reyes-Castillo, \& Pérez, 2011). Con respecto a Odontotaenius zodiacus se han registrado siete especies de Klinckowstroemia (Villegas-Guzmán, Francke, Pérez, \& Reyes-Castillo, 2012), mientras que para $O$. striatopunctatus tan sólo una de Diarthrophallidae (Schuster \& Summers, 1978). El presente estudio tiene como objetivo estimar la diversidad de especies de ácaros asociados a dos especies de Odontotenius en México y la distribución de estos en el cuerpo del pasálido. 


\section{MATERIALES Y MÉTODOS}

Obtención y procesamiento de muestras: Se examinaron pasálidos de Odontotaenius zodiacus y $O$. striatopunctatus depositados en la Colección Entomológica del INECOL de Xalapa (IEXA), recolectados en los estados de Hidalgo (13 marzo, 27 mayo 1978 y 12 octubre 1979) y Puebla (14 octubre 1979). Así como las recolectas de pasálidos realizadas por integrantes de las Colecciones Nacionales de Ácaros (CNAC) y Arácnidos (CNAN) del Instituto de Biología de la UNAM, procedentes de los estados de Hidalgo (28 abril, 22 y 23 septiembre 2006), Chiapas (3 mayo 2004, 7 y 11 abril 2005, 7 mayo 2006 y 9 agosto 2006), Veracruz (12 noviembre 2004, 23 y 30 septiembre 2006, 23 marzo 2007), Tamaulipas (septiembre 2004) y Tabasco (12 mayo 2006).

Cada uno de los pasálidos fue capturado en troncos en descomposición y colocados individualmente en frascos con alcohol etílico al $80 \%$. Los pasálidos fueron revisados, y los ácaros encontrados en las diferentes partes del cuerpo fueron depositados en frascos con alcohol al $80 \%$, algunos fueron procesados con lactofenol para aclararlos de 24 a 72 horas. Se montaron con líquido de Hoyer y se secaron en una estufa a una temperatura de $45^{\circ} \mathrm{C}$ durante 5-7 días para su posterior determinación a nivel específico. Los ácaros están depositados en la colección del Laboratorio de Acarología Dra. Isabel Bassols Batalla de la Escuela Nacional de Ciencias Biológicas, IPN y en La Colección Nacional de Ácaros (CNAC) del Instituto de Biología, UNAM. Los pasálidos en la Colección Entomológica del Instituto de Ecología de Xalapa (IEXA).

Se calculó la riqueza y abundancia específicas, estimando el índice de riqueza de Margalef ( $\left.\mathbf{S}^{\prime}\right)$, la diversidad de los ácaros asociados a cada uno de los pasálidos utilizando el índice de Shannon-Wiener $\left(\mathbf{H}^{\prime}\right)$ y con base al mismo la equidad $\left(\mathbf{E}_{\mathbf{H}}\right)$; además del índice de similitud de Jaccard (Moreno, 2001; Chao, Chazdon, Colwell, \& Shen, 2005; Bertolaccini, NuñesPérez, \& Tizado, 2012), con el fin de comparar las comunidades de ácaros en cada uno de los pasálidos y los estados, para ello se realizó un dendrograma con el programa Past versión 2.17c. Se calcularon curvas de acumulación de especies, basadas en el modelo de Clench (Moreno, 2001), con ayuda de los programas EstimateS (Versión 9.1.0) y Statistica (Statsoft) para medir la eficiencia del muestreo.

\section{RESULTADOS}

Se revisaron un total de 80 pasálidos pertenecientes al género Odontotaenius: 64 de $O$. zodiacus y 16 de $O$. striatopunctatus, recolectados en seis estados de la República Mexicana, correspondientes a 17 localidades (Cuadro 1). Se obtuvo un total de 1945 ácaros (396 우우,

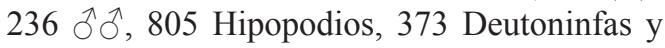
135 Tritoninfas), la mayor proporción encontrada correspondió a los estadios ninfales con 1314 ejemplares (68\%), los adultos sólo representaron un $32 \%$ del total (Cuadro 2). Se encontraron 42 especies pertenecientes a 13 familias de tres órdenes, la mayor riqueza fue registrada en la Diarthrophallidae (14 especies), seguida de Diplogyniidae y Euzerconidae con cinco especies cada una (Cuadros 3 y Cuadro 4).

Para Odontotaenius striatopunctatus, se contabilizaron 562 organismos (95 우, 34

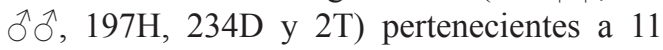
familias. La de mayor proporción fue Uropodidae (42\%), seguida de Histiostomatidae (26\%). Las familias menos abundantes fueron Digamasellidae, Laelapidae y Klinckowstroemiidae, con uno o dos ejemplares.

En cuanto a Odontotaenius zodiacus, se encontraron un total de 1383 ácaros (300 우우,

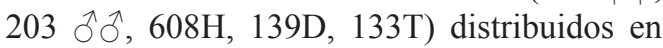
diez familias. Las familias más abundantes fueron Diartrophallidae y Acaridae con un $23 \%$ cada una, seguida de Histiostomatidae (21\%); en menor proporción encontramos a las familias Ascidae, Klinckowstroemiidae y Trematuridae (Cuadro 3 y Cuadro 4).

La especie más abundante fue Anoetus sp. con un $21.5 \%$, seguida de Sancassania sp. $(18.5 \%)$. Las que registraron la abundancia más baja $(0.05 \%)$ fueron: Abrotarsala 
CUADRO 1

Localidades de recolecta de los pasálidos del género Odontotaenius

TABLE 1

Collection localities of passalid of genus Odontotaenius

\begin{tabular}{|c|c|c|c|c|}
\hline Estado & Localidad & Altitud (m) & Coordenadas & Vegetación \\
\hline \multirow[t]{5}{*}{ Chiapas } & Reserva "La Lacandonia" & $159-204$ & $16^{\circ} 44.798^{\prime} \mathrm{N}$ y $91^{\circ} 00.757^{\prime} \mathrm{W}$ & Selva Alta Perennifolia \\
\hline & Centro de Convenciones & 209 & $17^{\circ} 29.204^{\prime} \mathrm{N}$ y $92^{\circ} 01.292^{\prime} \mathrm{W}$ & Selva Alta Perennifolia \\
\hline & "Ruinas de Palenque" & & & \\
\hline & Palenque & 97 & $17^{\circ} 29.073^{\prime} \mathrm{N}$ y $92^{\circ} 01.341^{\prime} \mathrm{W}$ & Selva Alta Perennifolia \\
\hline & Reserva Comunal "La Cruz" & 218 & $16^{\circ} 43.718^{\prime} \mathrm{N}$ y $90^{\circ} 55.852^{\prime} \mathrm{W}$ & Selva Alta Perennifolia \\
\hline \multirow[t]{6}{*}{ Hidalgo } & Crucero-Tianguistengo & 2080 & $20^{\circ} 40.413^{\prime} \mathrm{N}$ y $98^{\circ} 40.255^{\prime} \mathrm{W}$ & Bosque Encino \\
\hline & Acaxochitlán & $2100-2214$ & $\begin{array}{l}20^{\circ} 09^{\prime} 29.48^{\prime} \mathrm{N} \text { y } 98^{\circ} 11^{\prime} 59.41^{\prime \prime} \mathrm{W} ; \\
20^{\circ} 08^{\prime} 28.6^{\prime \prime} \text { y } 98^{\circ} 11^{\prime} 46.68^{\prime \prime} \mathrm{W}\end{array}$ & Bosque de Pino-Encino \\
\hline & Ejido Atepixco & 1972 & $20^{\circ} 35.466^{\prime} \mathrm{N}$ y $98^{\circ} 36.358^{\prime} \mathrm{W}$ & Bosque de Pino-Encino \\
\hline & Mojonera-Tizapán & 2019 & $20^{\circ} 38.107^{\prime} \mathrm{N}$ y $98^{\circ} 35.981^{\prime} \mathrm{W}$ & Bosque de Pino-Encino \\
\hline & Tlanchinol & 1555 & $21^{\circ} 01.003^{\prime} \mathrm{N}$ y $98^{\circ} 38.933^{\prime} \mathrm{W}$ & Bosque Mesófilo de Montaña \\
\hline & Zacualtipán & 1988 & $20^{\circ} 38^{\prime} 45.63^{\prime} \mathrm{N}$ y $98^{\circ} 39^{\prime} 16.89^{\prime \prime} \mathrm{W}$ & Bosque de Pino \\
\hline Puebla & Huauchinango & 2309 & $20^{\circ} 09^{\prime} 05.7^{\prime \prime} \mathrm{N}$ y $98^{\circ} 12^{\prime} 49.58^{\prime \prime} \mathrm{W}$ & Bosque Mesófilo de Montaña \\
\hline Tabasco & Finca Cholula & 51 & $18^{\circ} 17.010^{\prime} \mathrm{N}$ y $93^{\circ} 12.644^{\prime} \mathrm{W}$ & Selva Media Perennifolia \\
\hline Tamaulipas & Hidalgo & 322 & $24^{\circ} 14^{\prime} 09.07^{\prime \prime} \mathrm{N}$ y $99^{\circ} 25^{\prime} 39.49^{\prime \prime} \mathrm{W}$ & Bosque Mesófilo de Montaña \\
\hline \multirow[t]{4}{*}{ Veracruz } & Córdoba & 838 & $18^{\circ} 53^{\prime} 14.03 ” \mathrm{~N}$ y $96^{\circ} 55^{\prime} 23.02^{\prime \prime} \mathrm{W}$ & Bosque Mesófilo de Montaña \\
\hline & Soledad Atzompa & 2125 & $18^{\circ} 44.712^{\prime} \mathrm{N}$ y $97^{\circ} 40.967^{\prime} \mathrm{W}$ & Bosque de Pino-Encino \\
\hline & Viborillas & 2328 & $20^{\circ} 31.245^{\prime} \mathrm{N}$ y $98^{\circ} 29.923^{\prime} \mathrm{W}$ & Bosque de Pino-Encino \\
\hline & Tlacolulan & 1876 & $19^{\circ} 38.508^{\prime} \mathrm{N}$ y $96^{\circ} 59.650^{\prime} \mathrm{W}$ & Bosque de Pino-Encino \\
\hline
\end{tabular}

\section{CUADRO 2}

Número de ácaros por familias encontrados sobre dos especies de pasálidos del género Odontotaenius. Entre paréntesis el número de especies

\section{TABLE 2}

Mites number of families found on two passalids species of the genus Odontotaenius.

In brackets the number of species

\begin{tabular}{|c|c|c|c|c|c|}
\hline Orden & Suborden & Familia & O. striatopunctatus & O. zodiacus & Total \\
\hline \multirow[t]{10}{*}{ Mesostigmata } & \multirow[t]{6}{*}{ Monogynaspida } & Ascidae (3) & - & $2 \mathrm{D}$ & 2 \\
\hline & & Diarthrophallidae (14) & 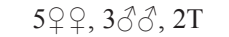 & 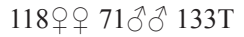 & 332 \\
\hline & & Digamasellidae (2) & $1 \mathrm{D}$ & $16 \mathrm{D}$ & 17 \\
\hline & & Laelapidae (1) & 19 & - & 1 \\
\hline & & Trematuridae (2) & $14 \mathrm{D}$ & $1 \mathrm{D}$ & 15 \\
\hline & & Uropodidae (3) & 219D & $123 \mathrm{D}$ & 342 \\
\hline & \multirow[t]{4}{*}{ Trigynaspida } & Diplogyniidae (5) & 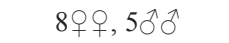 & $157 ㅇ ㅜ ㅇ, 128 \widehat{\jmath} \widehat{0}$ & 298 \\
\hline & & Euzerconidae (5) & $79 ㅇ ㅜ, 25 \hat{\jmath}$ & - & 104 \\
\hline & & Klinckowstroemiidae (4) & $1+, 10^{\pi}$ & 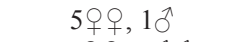 & 8 \\
\hline & & Megisthanidae (1) & - & 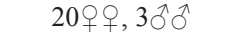 & 23 \\
\hline Trombidiformes & Prostigmata & Heterocheylidae (1) & 1 & - & 1 \\
\hline \multirow[t]{2}{*}{ Sarcoptiformes } & \multirow[t]{2}{*}{ Oribatida } & Acaridae (3) & $49 \mathrm{H}$ & $314 \mathrm{H}$ & 363 \\
\hline & & Histiostomatidae (1) & $148 \mathrm{H}$ & $291 \mathrm{H}$ & 439 \\
\hline Total & & 13 & 562 & 1383 & 1945 \\
\hline
\end{tabular}

$\mathrm{D}=$ deutoninfa, $\mathrm{H}$ = hipopodio, $\mathrm{T}$ = tritoninfa. (---) familias sin registro de ejemplares para el pasálido correspondiente. 
CUADRO 3

Distribución geográfica y zonas de fijación de las especies del suborden Monogynaspida asociadas a Odontotaenius zodiacus y O. striatopunctatus en México

TABLE 3

Geographical distribution and attachment zones of the species of the suborder Monogynaspida associated Odontotaenius zodiacus and $O$. striatopunctatus in Mexico

\begin{tabular}{|c|c|c|c|c|}
\hline Familia & Especie & Zonas de fijación & Huésped & Estado \\
\hline Ascidae & Sin determinar (s.d) & $\mathrm{Be}, \mathrm{Am}$ & O. zodiacus & Hgo. \\
\hline \multirow[t]{14}{*}{ Diarthrophallidae } & Abrotarsala arciformis & $\mathrm{Be}, \mathrm{Am}$ & O. zodiacus & Pue.*, Hgo.* y Ver.* \\
\hline & A. cuneiformis & $\mathrm{Be}$ & O. zodiacus & Hgo.** \\
\hline & A. obesa & C II y III, M, Am, Be, P & O. striatopunctatus y O. zodiacus & Hgo., Ver. y Chis.** \\
\hline & A. pyriformis & C II y III, Be, Am & O. zodiacus & Pue., Hgo., Ver.** \\
\hline & A. simplex & $\begin{array}{l}\text { C I, II y III, F I, Be y Am, } \\
\text { T III }\end{array}$ & O. striatopunctatus y O. zodiacus & $\begin{array}{l}\text { Hgo.*, Pue., Ver.*, } \\
\text { Chis* }\end{array}$ \\
\hline & Atrema nasica & C I y II, Be, Am, P, M & O. striatopunctatus y $O$. zodiacus & Hgo.*, Chis.* \\
\hline & Brachytremella sp. & $\mathrm{CI}, \mathrm{Be}$ & O. zodiacus & Hgo. $* *$ \\
\hline & Diarthrophallus aurosus & $\begin{array}{l}\text { Ca, C II y III, T II, Be, } \\
\text { F I, Am }\end{array}$ & O. zodiacus & Hgo* y Ver. \\
\hline & D. cartwrighti & $\mathrm{Be}, \mathrm{Am}$ & O. zodiacus & Hgo.** \\
\hline & D. crinatus & C III, T II, Be, P & O. zodiacus & Hgo.** \\
\hline & D. fulvastrum & $\mathrm{Be}$ & O. zodiacus & Hgo.* \\
\hline & D. quercus & $\begin{array}{l}\text { C I, II y III, Am, Be, } \\
\text { T I, F I, Ca, P }\end{array}$ & O. striatopunctatus y O. zodiacus & $\begin{array}{l}\text { Hgo., Ver.*, Chis., } \\
\text { Pue.* }\end{array}$ \\
\hline & Lombardiniella sp. & $\mathrm{Be}$ & O. zodiacus & Hgo.** \\
\hline & Tenuiplanta crossi & $\mathrm{P}$ & O. zodiacus & Hgo., Ver.* \\
\hline \multirow[t]{2}{*}{ Digamasellidae } & Dendrolaelaps sp. & Be, C I y III, Mt & O. striatopunctatus y $O$. zodiacus & Hgo., Chis. \\
\hline & Digamasellus sp. & Mt, C III, P, Be, Am & O. zodiacus & Hgo., Ver. \\
\hline Laelapidae & Cosmolaelaps sp. & $\mathrm{Be}$ & O. striatopunctatus & Chis. \\
\hline \multirow[t]{2}{*}{ Trematuridae } & Nenteria sp. & F I & O. striatopunctatus & Tab. \\
\hline & Trichouropoda ovalis *** & C I & O. zodiacus & Hgo. \\
\hline \multirow[t]{3}{*}{ Uropodidae } & Uroobovella californiana & C III, Ca, Tro, ++ & O. striatopunctatus y $O$. zodiacus & Chis.*, Tamps.* \\
\hline & Uropoda orbicularis & F I, C, Tro, P, ++ & O. striatopunctatus y $O$. zodiacus & Chis.*, Hgo.*, Tab.* \\
\hline & Uropoda sp. & C I, II y III, M, Ca, H, ++ & O. striatopunctatus y $O$. zodiacus & Chis., Hgo., Ver. \\
\hline
\end{tabular}

$\mathbf{A m}=$ alas membranosas, $\mathbf{B e}=$ bajo los élitros, $\mathbf{C}=$ coxas, $\mathbf{H}=$ húmero, Tro = trocánter, $\mathbf{M}=$ mentón, $\mathbf{M t}=$ metasternón, $\mathbf{P}=$ proesternón, $\mathbf{F}=$ fémur, $\mathbf{C a}=$ cabeza, $\mathbf{T}=$ tibia. $*$ Nuevo registro estatal. $* *$ Nuevos registros para el país. $* * *$ Especie registrada por primera vez sobre pasálidos. ++ Organismos encontrados en el alcohol.

cuneiformis, Brachytremella sp., Diarthrophallus cartwrighti, D. fulvastrum, Cosmolaelaps sp., Nenteria sp., Trichodiplogynium carlosi, Euzercon brachys, Klinckowstroemia pennula, K. franckei, Heterocheylus proximus, Histiogaster sp. y Tyrophagus putrescentiae.

Doce de las especies encontradas fueron registradas por primera vez sobre pasálidos mexicanos: Abrotarsala cuneiformis, A. obesa, A. pyriformis, Brachytremella sp., Diarthrophallus cartwrighti, D. crinatus, Lombardiniella sp., Trichodiplogynium carlosi, T. sahlbergi, T. hirsutum y Trichodiplogynium sp. (Cuadro 3 y Cuadro 4).
Distribución de los ácaros en el cuerpo del pasálido: Fueron encontrados en su mayoría en zonas protegidas del pasálido como bajo los élitros $(50 \%)$ y bajo las alas membranosas (34\%) y en menor proporción en la región coxal y de la cabeza del pasálido (21\%). De acuerdo al tipo de foresia que realizan los ácaros se pueden clasificar en tres grupos: las familias que realizan foresia estrictamente activa: Heterocheylidae, Histiostomatidae, Euzerconidae, Klinckowstroemiidae, Megisthanidae, Trematuridae y Uropodidae al encontrarse en zonas expuestas del pasálido (Cuadro 3 y Cuadro 4). Ascidae y Laelapidae realizan foresia 
CUADRO 4

Distribución geográfica y zonas de fijación de las especies de los subórdenes Trigynaspida, Prostigmata y Oribatida asociadas a Odontotaenius zodiacus y $O$. striatopunctatus en México

TABLE 4

Geographical distribution and attachment zones of the species suborders Trigynaspida, Prostigmata and Oribatida associated Odontotaenius zodiacus and $O$. striatopunctatus at Mexico

\begin{tabular}{|c|c|c|c|c|}
\hline Familia & Especie & Zonas de fijación & Huésped & Estado \\
\hline \multirow{5}{*}{ Diplogyniidae } & Trichodiplogynium carlosi & ++ & O. zodiacus & $\mathrm{Hg}_{0}{ }^{* *}$ \\
\hline & T. hirsutum & $\mathrm{C}$ & O. striatopunctatus & Chis, ${ }^{* *}$ \\
\hline & T. sahlbergi & C I, II y III, Ca, Am, M, P, Be, T I & O. zodiacus & Hgo.,Ver.** \\
\hline & Trichodiplogynium sp. & M, C II y III, Am & O. zodiacus & $\mathrm{Hg}_{0}{ }^{* *}$ \\
\hline & Tridiplogynium inexpectatum & $\mathrm{C}$ I, Be & O. striatopunctatus & Chis.** \\
\hline \multirow[t]{5}{*}{ Euzerconidae } & Euzercon anatonon & ++ & O. striatopunctatus & Tab.* \\
\hline & E. brachys & ++ & O. striatopunctatus & Chis.* \\
\hline & E. dolichos & C II y III, Ca & O. striatopunctatus & Chis.*, Tab.*, Ver. \\
\hline & E. latus & $\mathrm{C}$ & O. striatopunctatus & Chis. \\
\hline & E. subtermion & C III, P & O. striatopunctatus & Chis.*, Tab.* \\
\hline \multirow[t]{4}{*}{ Klinckowstroemiidae } & Klinckowstroemia bifurcata & C I y II, Ca, P & O. zodiacus & Hgo. \\
\hline & K. pennula & ++ & O. zodiacus & Ver.* \\
\hline & K. franckei & F I, M, ++ & O. zodiacus & Hgo. \\
\hline & K. simplisetosa & $\mathrm{Ca}$ & O. striatopunctatus & Chis.* \\
\hline Megisthanidae & Megisthanus floridanus & Ta III, ++ & O. zodiacus & Hgo. \\
\hline Heterocheylidae & Hetecheylus proximus & C III & O. striatopunctatus & Tamps. \\
\hline \multirow[t]{3}{*}{ Acaridae } & Histiogaster $s p .^{* * *}$ & $\mathrm{Am}$ & O. zodiacus & Hgo. \\
\hline & Sancassania sp. & C I & O. striatopunctatus y $O$. zodiacus & Hgo.*, Ver. \\
\hline & Tyrophagus putrescentiae*** & C III & O. striatopunctatus & Tamps. \\
\hline Histiostomatidae & Anoetus sp. & $\mathrm{P},++$ & O. striatopunctatus y O. zodiacus & Ver. \\
\hline
\end{tabular}

$\mathbf{A m}=$ alas membranosas, $\mathbf{B e}=$ bajo los élitros, $\mathbf{C}=$ coxas, $\mathbf{T a}=$ tarso, $\mathbf{M}=$ mentón, $\mathbf{P}=$ proesternón, $\mathbf{F}=$ fémur, $\mathbf{C a}=$ cabeza, $\mathbf{T}=$ tibia. *Nuevo registro estatal. **Nuevos registros para el país. ***Especie registrada por primera vez sobre pasálidos. ++ Organismos encontrados en el alcohol.

pasiva (Cuadro 3), al sólo ser localizadas bajo los élitros, alas membranosas y hendiduras del pasálido. Mientras que Acaridae, Diarthrophallidae, Digamasellidae y Diplogyniidae parecen presentar una alternancia entre foresia activa y pasiva al ubicarse tanto en las zonas protegidas como en las expuestas (Cuadro 3 y Cuadro 4).

Se observó la hiperforesia en ejemplares de Uropoda orbicularis, los que se encontraron anclados a una hembra de Megisthanus floridanus que a su vez estaba sobre el pasálido O. striatopunctatus.

Se obtuvieron un total de 124 hembras con huevos (34\% con respecto al total de ácaros), representando a cuatro familias (Diarthrophallidae, Diplogyniidae, Euzerconidae y Klinckowstroemiidae). La que presentó mayor número de hembras grávidas fue Diplogyniidae con un $71 \%$, seguida de Euzerconidae con un
$20 \%$; los klinckowstroémidos sólo tuvieron un $1 \%$ de hembras grávidas.

Riqueza, diversidad y eficiencia de muestreo: La mayor abundancia y riqueza se registró para los ejemplares de Odontotaenius zodiacus, el cual contó con 1383 ácaros, agrupados en 30 especies, en comparación con $O$. striatopunctatus que contó con 562 organismos en donde sólo se registraron 22 especies.

Las curvas de acumulación para ambas especies de pasálidos, se realizaron con el fin de calcular la efectividad en el muestreo. Para $O$. striatopunctatus se obtuvo una efectividad del $65 \%$, la de $O$. zodiacus muestró un $92 \%$. Para ambos pasálidos la curva de acumulación presenta una distribución logarítmica con ajuste en la ecuación de la pendiente de Clench. El coeficiente de determinación $\left(\boldsymbol{R}^{2}\right)$ en ambas 
curvas es cercano a uno, lo que indica un buen ajuste del modelo; sin embargo, no es posible observar una asíntota ya que el número de muestras consideradas no fue suficiente para una inflexión en la curva (Fig. 1 y Fig. 2).
En el índice de riqueza de especies de Margalef se obtiene que el valor calculado para $O$. striatopunctatus (3.34) fue menor que el de O. zodiacus (4.05) (Cuadro 5), debido a que 22 especies encontradas se asociaron a $O$.

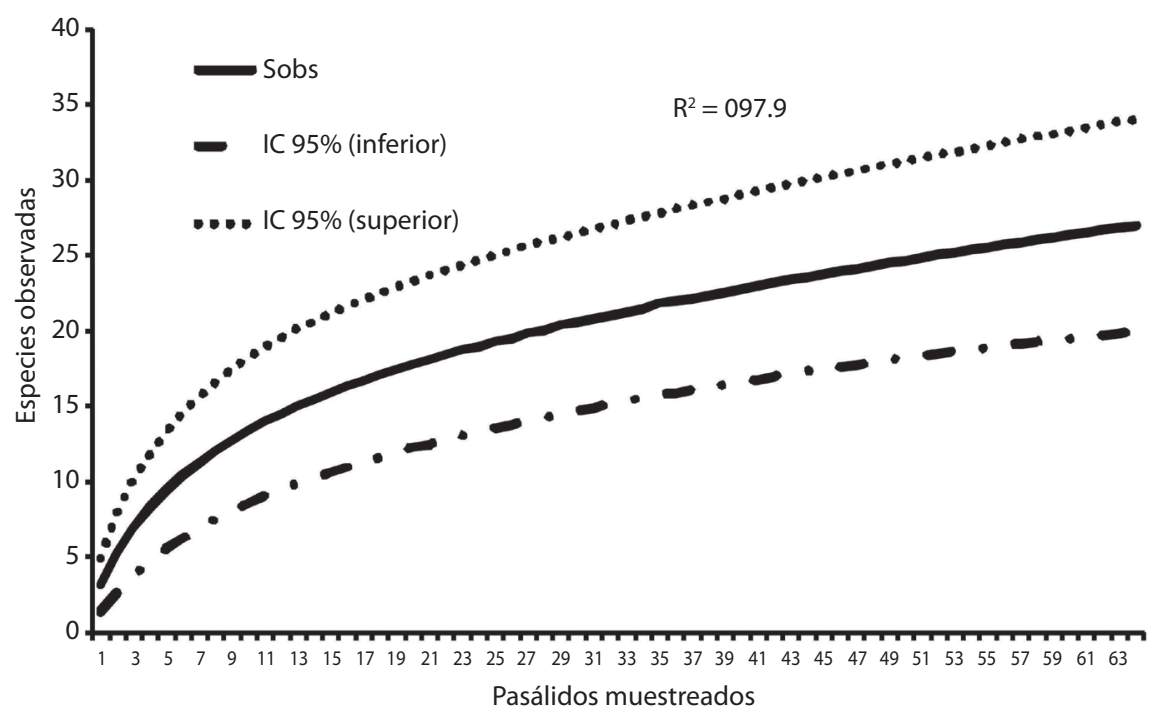

Fig. 1. Curva de acumulación de especies para O. striatopunctatus.

Fig. 1. Species accumulation curve for O. striatopunctatus.

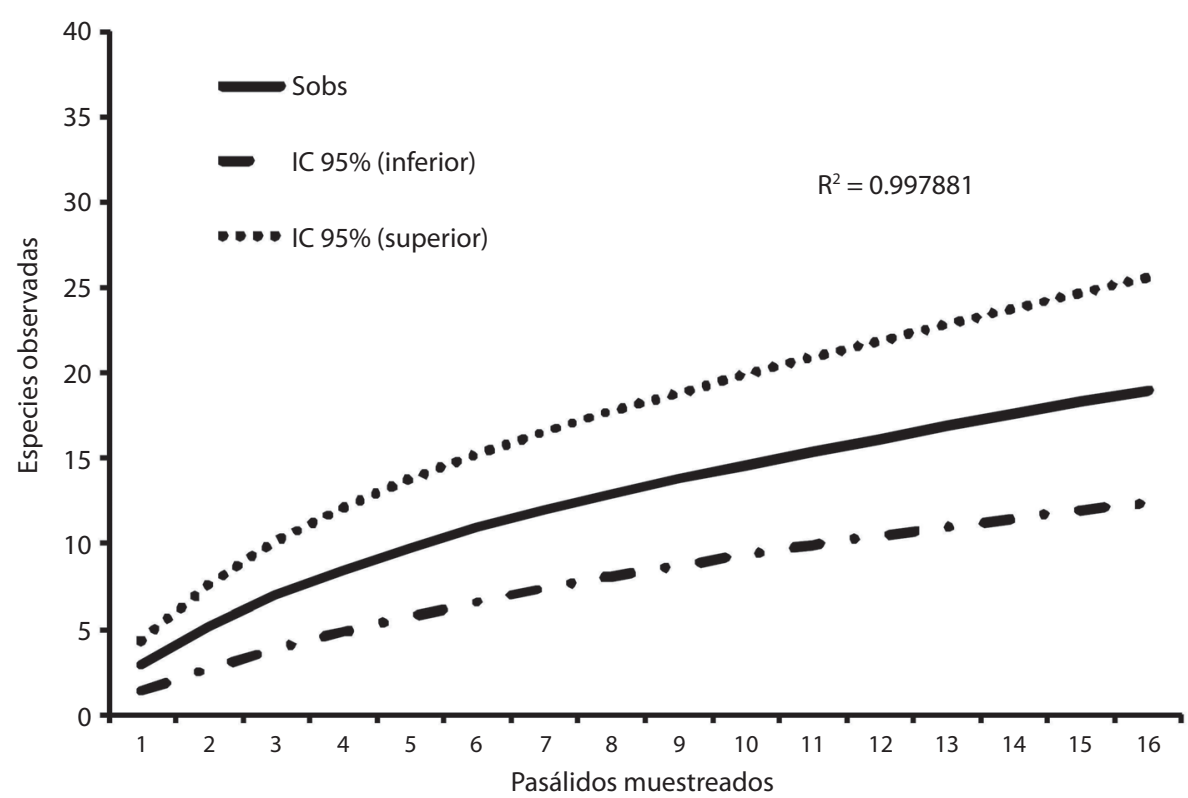

Fig. 2. Curva de acumulación de especies para $O$. zodiacus.

Fig. 2. Species accumulation curve for $O$. zodiacus. 
CUADRO 5

Índices de diversidad y riqueza para las dos especies de pasálidos

TABLE 5

Diversity and richness indexes for the two species passalids

\begin{tabular}{|c|c|c|}
\hline \multirow{2}{*}{ Índice } & \multicolumn{2}{|c|}{ Pasálido } \\
\hline & O. striatopunctatus & O. zodiacus \\
\hline Número de muestras & 16 & 64 \\
\hline Abundancia absoluta $(\mathbf{N})$ & 562 & 1383 \\
\hline Riqueza de Margalef ('S') & 3.34 & 4.05 \\
\hline Índice de Shannon-Wiener $\left(\mathbf{H}^{\prime}\right)$ & 1.94 & 2.2 \\
\hline Equidad $\left(E_{H}\right)$ & 0.63 & 0.64 \\
\hline Similitud de Jaccard $\left(\mathbf{I}_{\mathbf{j}}\right)$ & \multicolumn{2}{|c|}{$0.24(24 \%)$} \\
\hline Especies asociadas $\left(\mathbf{S}^{\prime}\right)$ & 22 & 30 \\
\hline Especies compartidas $(\mathbf{C})$ & \multicolumn{2}{|c|}{10} \\
\hline
\end{tabular}

striatopunctatus y 30 a $O$. zodiacus. Se obtuvo un índice de diversidad de Shannon-Wiener mayor en $O$. zodiacus $\left(\mathbf{H}^{\prime}=2.2\right)$ que en $O$. striatopunctatus $\left(\mathbf{H}^{\prime}=1.94\right)$ (Cuadro 5). La equidad $\left(\mathbf{E}_{\mathbf{H}}\right)$ encontrada es similar para ambos huéspedes $O$. zodiacus $\left(\mathbf{E}_{\mathbf{H}}=0.64\right)$ y $O$. striatopunctatus $\left(\mathbf{E}_{\mathbf{H}}=0.63\right)$ (Cuadro 5).

El análisis de agrupamiento con el índice de similitud de Jaccard, tomando en cuenta la abundancia de especies entre los dos pasálidos y la abundancia de las mismas por estado, mostró dos grupos principales (Fig. 3): el grupo A con el $31 \%$ de similitud se conforma por 12 especies y el $\mathbf{B}$ con las 30 restantes (41\% de similitud). El grupo A se caracteriza por especies recolectadas en los estados de Chiapas, Veracruz, Tabasco y Tamaulipas a altitudes desde los 51 a los $838 \mathrm{msnm}$, todas en asociación con $O$. striatopunctatus. Once de las especies sólo se registraron en un estado a excepción de Euzercon dolichos presente en tres estados, dicha especie se une al grupo por el huésped y no por la localidad. Nenteria sp. se queda en un grupo debido al número de ejemplares encontrados en comparación con el resto de las especies, al igual que Heterocheylus proximus y Tyrophagus putrescentiae, contó con un sólo ejemplar, pero a diferencia de estos, es el único con un ejemplar encontrado en Tabasco.

En el grupo B se pueden distinguir dos subgrupos: B1 con una similitud del $56 \%$ se conforma por 20 especies en asociación con
O. zodiacus de los estados de Hidalgo, Puebla y Veracruz que a su vez se subdivide en cuatro grupos. Klinckowstroemia pennula, se quedó sólo al tratarse de una especie con un sólo ejemplar localizado en Veracruz. El grupo más numeroso se conforma por 13 especies del estado de Hidalgo. La similitud más alta en el subgrupo es del $73 \%$ y se da entre cuatro especies localizadas en los estados de Hidalgo y Veracruz. El subgrupo B2 se cuenta por las 10 especies compartidas por ambos pasálidos de los estados de Chiapas, Hidalgo, Puebla y Veracruz con una similitud del $69 \%$. La similitud más alta en el subgrupo la conforman las especies Uropoda sp. y U. orbicularis (83\%), las que estuvieron presentes en cinco de los seis estados muestreados, con una abundancia relativa del $8.6 \%$ en $O$. zodiacus y un $42 \%$ con respecto a $O$. striatopunctatus.

\section{DISCUSIÓN}

El pasálido $O$. zodiacus se considera nuevo huésped de ácaros foréticos, registra la mayor riqueza encontrada hasta el momento con 30 especies (12 familias), seguido de O. striatopunctatus con 22 especies (11 familias). Estudios previos han reportado a Popilius disjunctus con 16 especies (nueve familias) (Delfinado \& Baker, 1975), Passalus cornutus con 13 especies (ocho familias) (Pearse, Patterson, Rankin, \& Wharton, 1936) y Passalus cognathus con ocho especies (ocho familias) 


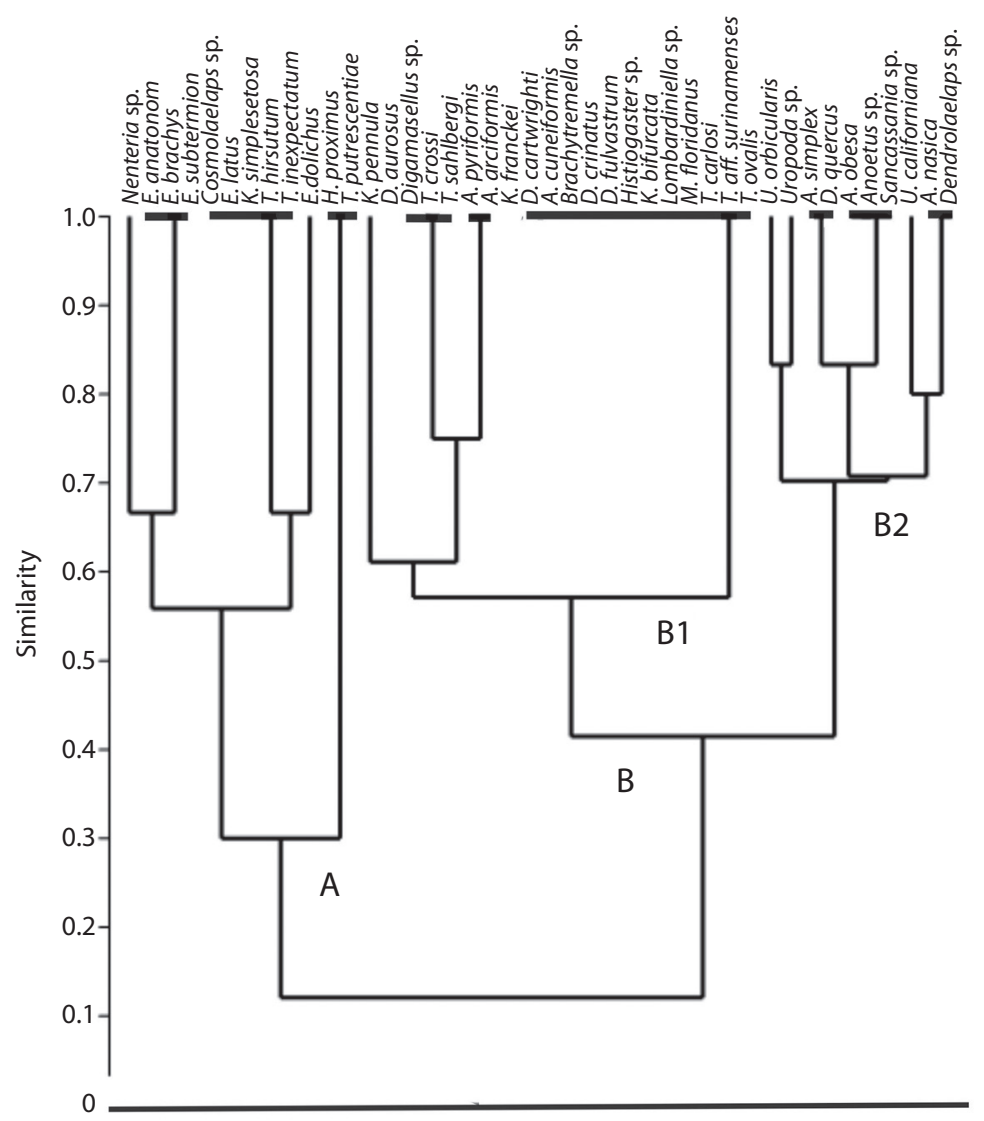

Fig. 3. Agrupamiento de especies de ácaros por índice de similitud de Jaccard.

Fig. 3. Grouping mite species by Jaccard similarity index.

(Villegas-Guzmán, Pérez, \& Reyes-Castillo, 2008). En cuanto a la distribución de los ácaros sobre el cuerpo del pasálido, lo reportado coincide con los trabajos de Schuster y Summers (1978), Wallace (1986) y Villegas-Guzmán et al. (2008).

Se distinguen dos principales grupos de ácaros en los pasálidos estudiados, aquellos que realizan foresia activa y los forontes pasivos. Los primeros son aquellos que se fijan al huésped a través de las uñas y carúnculas de las patas que les sirven como estructuras de succión para adherirse a las partes lisas del pasálido (Hunter, 1993a); aquí se incluyen a los euzercónidos y heteroquélidos (Hunter \& Davis, 1965; Hunter, 1993a). En el caso de los estadios ninfales en uropódidos y trematúridos han desarrollado un pedicelo anal que les permite fijarse a las zonas expuestas, es común encontrarlos en la superficie ventral en áreas gulares y coxales (Binns, 1981). Los histiostomátidos son comunes en los pasálidos y al igual que los acáridos juveniles cuentan con una placa suctorial en el área anal que les permite la unión al huésped, principalmente se ubican en las coxas III (Hunter, 1993a). Los klinckowstroémidos se encontraron en las coxas, cabeza, proesternón y alcohol, lo cual concuerda con los registros previos (Villegas-Guzmán et al., 2012). Los adultos de Megisthanus floridanus son los forontes, estos cuentan con carúnculas en las patas para anclarse a zonas expuestas de su huésped (Butler \& Hunter, 1968), además, por su tamaño y forma del cuerpo es poco 
probable encontrarlos en las zonas protegidas del cuerpo del pasálido.

Los forontes pasivos presentan un cuerpo aplanado dorsoventralmente y son muy pequeños, lo que les facilita su ubicación en cavidades del cuerpo. La foretomorfa de los áscidos cumplen con dichas características, al igual que Cosmolaelaps sp., también utilizan sus quelíceros para sujetarse a las sedas del huésped, se les ha localizado en las sedas de las patas I (Hunter \& Mollin, 1964). Los acáridos, diplogínidos, digamasélidos y diartrofálidos, presentan estructuras de succión y su morfología les permite transportarse en zonas protegidas del cuerpo del pasálido; los diartrofálidos se alimentan de las secreciones o residuos de su huésped (Hunter, 1993a). Los hipopodios de los acáridos presentan una membrana suctorial localizada en la placa anal y son aplanados dorsoventralmente, pueden fácilmente situarse bajo las alas, los élitros o cualquier sitio protegido en el cuerpo del pasálido. La mayoría de los diplogínidos fueron encontrados en zonas expuestas como las coxas y tibias de las patas, cabeza, proesternón y mentón. A pesar de ello, Trichodiplogynium sahlbergi y Tridiplogynium inexpectatum también fueron localizados bajo los élitros de $O$. zodiacus y $O$. striatopunctatus, respectivamente. Tridiplogynium se ha localizado en los húmeros de Passalus chingaencis y Petrejoides subrecticornis (Cómbita-Heredia, 2006). Dendrolaelaps sp. y Digamasellus sp. se ubicaron bajo los élitros, además de zonas expuestas como las coxas, metasternón y proesternón (Cuadro 3). Dendrolaelaps ha sido registrado bajo los élitros, proesternón, mesoesternón, metaesternón, trocánter III y en los urosternitos (Hunter, 1993a; Cómbita-Heredia, 2006). Los diartrofálidos se extrajeron principalmente bajo los élitros y las alas membranosas, además en zonas expuestas como lo son las coxas, tibias, proesternón y mentón, lo que coincide con los datos obtenidos por Schuster y Summers (1978) y Pearse et al. (1936).

Con base en la clasificación de los tipos de foresia propuesta por Athias-Binche (1994), los ejemplares de Ascidae y Tyrophagus putrescentiae son forontes ocasionales, los áscidos son depredadores de nemátodos y de otros ácaros que comúnmente se encuentran en las galerías de los escarabajos (Moser, 1995). T. putrescentiae se alimenta de hongos degradadores de celulosa como son algunos ascomicetos de los géneros Chaetomium, Fusarium, Alternaria, Cladosporium, Curvularia y Nigrospora (Sinha \& Wallace, 1966; Heredia-Abarca, 2003; Mauri \& Bischoff De Alzuet, 1966).

Las familias Histiostomatidae, Uropodidae, Trematuridae y Digamasellidae además de las especies Histiogaster sp. y Sancassania sp., se consideran como forontes facultativos que pasan todo su ciclo en vida libre, pero con la capacidad de desarrollar foretomorfas influenciados por cambios en el microclima (Binns, 1981). Los diplogínidos y euzercónidos caen también en esta categoría, sólo los adultos se dispersan sobre el pasálidos, el resto de los estadios vive en las galerías, hojarasca y suelo al ser depredadores de pequeños artrópodos (Hunter \& Davis 1965). Los trigináspidos, son organismos generalistas (depredadores, fungívoros o carroñeros) (Seeman \& Walter 1997).

La presencia de hembras grávidas es una de las estrategias comúnmente observadas en varias especies, con esto aseguran la colonización del nuevo sitio al que se desplaza. Hunter (1993a) sugiere que cuando las hembras se dispersan sin el macho son partenogenéticas, pero cuando ambos sexos lo hacen, la cópula se lleva a cabo después de la dispersión.

Uropodidae presentó la más amplia distribución al registrarse en cinco estados: Chiapas, Hidalgo, Veracruz, Tabasco y Tamaulipas, a altitudes desde los 51 a los 2214 msnm. Ejemplares de Uropoda sp. y Uroobovella californiana han sido reportados en localidades de Chiapas, en altitudes desde 150 a los 700 msnm (Villegas-Guzmán et al., 2008), lo que coincide con el intervalo altitudinal de las recolectas realizadas para el estado de Chiapas, Veracruz y Tamaulipas. Uropoda orbicularis se registra en altitudes superiores a los 2000 msnm; su amplia distribución se debe probablemente a que no sólo se asocia a pasálidos 
sino que es uno de los uropódidos foréticos más comunes que pueden habitar en el suelo, plantas, aves y nidos de mamíferos (Bajerlein \& Przewozny, 2005).

La diversidad calculada es mayor en $O$. zodiacus al igual que la equidad, la cual se ve reflejada en la abundancia de las especies mayor representadas. En asociación con $O$. zodiacus las tres especie con mayor abundancia son: Sancassania sp. (22.6\%), Anoetus sp. $(21.3 \%)$ y Trichodiplogynium sahlbergi (20.1\%) acumulando más del 60\%. Las familias Ascidae y Megisthanidae sólo se encontraron sobre $O$. zodiacus. El género Megisthanus es cosmopolita, $M$. floridanus se ha reportado para Norteamérica (Butler \& Hunter, 1968). En O. striatopunctatus las especies más abundantes son: Uropoda sp. (29.9\%) y Anoetus sp. $(22.4 \%)$. Las familias Euzerconidae, Heterocheylidae y Laelapidae sólo se encontraron en $O$. striatopunctatus, estos grupos se han registrado en zonas tropicales de los estados de Chiapas, Veracruz y Tabasco (Funk, 1980; Hunter \& Rosario, 1989; Hunter \& Rosario, 1991)

La diferencia en la composición de especies de ácaros entre los pasálidos estudiados puede deberse a dos factores importantes: primero, al sesgo en el muestreo ya que no se cuenta con el mismo número de ácaros para cada pasálido y, segundo, a la distribución geográfica de cada uno, O. striatopunctatus se distribuye ampliamente desde Tamaulipas hasta la Península de Yucatán, a lo largo de la Sierra Madre Oriental y se desplazada a los estados de Oaxaca y Guerrero (Reyes-Castillo et al., 2006), incluso la especie se ha reportado en zonas de manglar en el estado de Tabasco (Castillo \& Reyes-Castillo, 2003); en cambio $O$. zodiacus se encuentra limitada la zona central de la sierra (Hidalgo, Puebla y Veracruz) (Fig. 3) (Castillo \& Reyes-Castillo, 2003).

En Veracruz se obtuvieron ambas especies de pasálidos, sin embargo, no coexisten en las mismas localidades, O. striatopunctatus se restringió a Córdoba únicamente en donde la altitud es menor a los $900 \mathrm{~m}$, mientras que $O$. zodiacus se registra en las localidades de Viborillas, Tlacolulan y Soledad Atzompa a altitudes superiores a los $2000 \mathrm{msnm}$ (Cuadro 1).

Con base en los resultados de efectividad de muestreo y debido al sesgo en el número de muestras comparadas se sugiere nuevas recolectas de O. striatopunctatus en los estados de Nuevo León, San Luis Potosí, Oaxaca, Guerrero, Yucatán y Quintana Roo, así como recolectas para ambos pasálidos en el estado de Puebla.

\section{AGRADECIMIENTOS}

A Tila María Pérez Ortiz de la Colección Nacional de Ácaros (CNAC) y a Oscar Francke de la Colección Nacional de Arácnidos (CNAN) del Instituto de Biología de la UNAM. A Pedro Reyes-Castillo de la Colección de Entomológica del Instituto de Ecología de Xalapa (IEXA) por el préstamo de los pasálidos para la realización del presente trabajo. A Griselda Montiel Parra por sus sugerencias al manuscrito. A dos revisores anónimos por sus sugerencias al manuscrito.

\section{RESUMEN}

Los ácaros se relacionan con pasálidos, estableciendo una asociación temporal para transportarse (foresia). Se conocen más de 200 especies de ácaros forontes de pasálidos. El objetivo del estudio fue conocer la diversidad de ácaros asociados a dos especies de Odontotaenius, así como determinar sus sitios de fijación. Se recolectaron pasálidos de troncos en descomposición en seis estados de México. Se encontraron 80 pasálidos con ácaros (64 de O. zodiacus y 16 de $O$. striatopunctatus), en total 1945 ácaros de 13 familias, distribuidos en 42 especies. En $O$. striatopunctatus se encontraron 562 ácaros (95 우우, 34

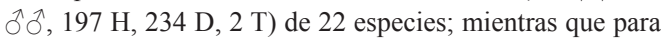
O. zodiacus, 1383 organismos (300 우, 204 ふैगे, $608 \mathrm{H}$ $139 \mathrm{D}, 133 \mathrm{~T})$ de 30 especies. La mayoría se encontraron en las coxas, bajo los élitros y las alas membranosas desarrollando foresia pasiva. La efectividad del muestreo fue mayor en $O$. zodiacus, lo que se refleja en los índices de diversidad y similitud. El índice de Shannon-Wiener, muestra una mayor diversidad en $O$. zodiacus $\left(\mathbf{H}^{\prime}=2.2\right)$ que en $O$. striatopunctatus $\left(\mathbf{H}^{\prime}=1.94\right)$. Odontotaenius es el género que tiene más especies de ácaros asociados y se registran por vez primera 12 especies: Abrotarsala cuneiformis, A. obesa, A. pyriformis, Brachytremella sp., 
Diarthrophallus cartwrighti, D. crinatus, Lombardiniella sp., Trichodiplogynium carlosi, T. sahlbergi, T. hirsutum y Trichodiplogynium sp.

Palabras clave: ácaros, diversidad, coleópteros pasálidos, nuevos registros, distribución.

\section{REFERENCIAS}

Amat-García, D., Blanco E., \& Reyes-Castillo, P. (2004). Lista de especies de los escarabajos pasálidos (Coleoptera: Passalidae) de Colombia. Biota Colombiana, 5, 173-182.

Athias-Binche, F. (1994). La phorésie chez les acariens: Aspects adaptatifs et Evolutifs. París, Francia: Editions du Castillet.

Bajerlein, D., \& Przewozny, M. (2005). Coprophagous hydrophilid beetles (Coleoptera: Hydrophilidae) as carriers of phoretic deutonymphs of Uropoda orbicularis (Acari: Mesostigmata) in Poland. European Journal of Entomology, 102, 119-122.

Bertolaccini, I., Núñes-Pérez, E., \& Tizado, E. J. (2012). Diversidad y proporción sexual de Syrphidae en cultivos de leguminosas y plantas espontáneas, en León (España). Revista de Ciencias Agrarias, 35, 99-107.

Binns, E. S. 1981. Some functional aspects of phoresy in mites. Biological Reviews, 57, 573-620.

Butler, L., \& Hunter, P. E. (1968). Redescription of Megisthanus floridanus with observations on its biology (Acarina: Megisthanidae). The Florida Entomologist, 51, 187-197.

Castillo, M., Rivera-Cervantes, L. E., \& Reyes-Castillo, P. (1988). Estudio sobre los Passalidae (Coleoptera: Lamellicornia) de la Sierra de Manantlán, Jalisco, México. Acta Zoológica Mexicana (n.s.), 30, 1-20.

Castillo, M. L. \& Reyes-Castillo, P. (2003). Los Passalidae: Coleópteros tropicales degradadores de troncos de árboles muertos. In J. Álvarez-Sánchez \& E. NaranjoGarcía (Eds.), Ecología del Suelo de la Selva Tropical Húmeda de México (pp. 237-262). Xalapa, Veracruz, México: Instituto de Ecología, A.C. Instituto de Biología, Facultad de Ciencias UNAM.

Chao, A., Chazdon, R. L., Colwell, R. K., \& Shen, T. (2005). Un nuevo método estadístico para la evaluación de la similitud en la composición de especies con datos de incidencia y abundancia. In G. Halffter, J. Soberón, P. Koleff, \& A. Melic (Eds.), Sobre Diversidad Biológica. El significado de las Diversidades Alfa, Beta y Gamma (pp. 85-95). Recuperado de http://viceroy.eeb.uconn.edu/estimates/EstimateSPages/EstSUsersGuide/References/ChaoEtA12005Sp. pdf

Cómbita-Heredia, J. O. (2006). Ácaros (Arachnida: Acari) asociados a escarabajos pasálidos (Coleoptera:
Passalidae) en Colombia. Acta Biológica Colombiana, 11, 1-11.

Delfinado, M. D., \& Baker, E. W. (1975). Mites (Acarina) associated with Popilius disjunctus (Illiger) (Coleoptera: Passalidae) in Eastern United States. Journal of the New York Entomological Society, 83, 49-59.

Funk, R. C. (1980). Generic revision of the family Euzerconidae (Mesostigmata: Celaenopsioidea) with description of seven new genera. International Journal of Acarology, 6, 313-349.

Heredia-Abarca, G. (2003). Los hongos microscópicos en la descomposición de las hojas. In J. Álvarez-Sánchez \& E. Naranjo-García (Eds.), Ecología del Suelo de la Selva Tropical Húmeda de México (pp. 263-273). Xalapa, Veracruz, México: Instituto de Ecología, A.C. Instituto de Biología, Facultad de Ciencias, UNAM.

Hunter, P. E., \& Mollin, K. (1964). Mites associated with the Passalus beetle. I. life stages and seasonal abundance of Cosmolaelaps passali n. sp. (Acarina: Laelapidae). Acarología, 6, 247-256.

Hunter, P. E., \& Davis, R. (1965). Mites associated with the passalus beetle III. Life stages and observations on biology of Euzercon latus (Banks) (Acarina: Euzerconidae). Acarología, 6, 247-256.

Hunter, P. E., \& Rosario, R. M. T. (1989). Two new genera and species of Euzerconidae (Mesostigmata: Celaenopsoidea) with a key to New World genera. Journal of Entomological Science, 24, 241-251.

Hunter, P. E., \& Rosario, R. M. T. (1991). New species of Euzercon Berlese from Mexico with a key to the New World species (Acarina: Mesostigmata: Euzerconidae). Acarología, 32, 205-216.

Hunter, P. E. (1993a). Mites associated with New World passalid beetles (Coleoptera: Passalidae). Acta Zoológica Mexicana (n.s.), 58, 1-37.

Hunter, P. E. (1993b). Two new genera, Crassoseta and Brachysternopsis, of the family Diplogyniidae (Acarina, Mesostigmata, Trigynapsida). Zoologica Scripta, 22, 91-99.

Mauri, R. \& Bischoff de Alzuet, A. (1966). Presencia de Tyrophagus putrescentiae (Scharank) (Sarcopt.Acaridae) en laboratorios. Revista del Museo de la Plata (n. s.), Sección Zoología, 9, 21-24.

Moreno, C. E. (2001). Métodos para medir la biodiversidad. Zaragoza: MyT-Manuales y Tesis SEA.

Moser, J. C. (1995). Mites associated with forest insects. Monroe, Oregon: Willamette Institute for Biological Control, Inc.

Paracer, S., \& Ahmadjian, V. (2000). Symbiosis. An introduction to biological associations ( $2^{\mathrm{a}}$ edition). Oxford: Oxford University Press. 
Pearse, A. S., Patterson, M. T., Rankin, J. S., \& Wharton, G. W. (1936). The ecology of Passalus cornutus Fabricius, a beetle which lives in rotting logs. Ecological Monographs, 6, 455-490.

Reyes-Castillo, P., \& Halffter, G. (1984). La estructura social de los Passalidae (Coleoptera: Lamellicornia). Folia Entomológica Mexicana, 61, 49-72.

Reyes-Castillo, P. (2000). Coleoptera Passalidae de México. In F. Martín-Piera, J. J. Morrone, \& A. Melic (Eds.), Hacia un Proyecto CYTED para el Inventario y Estimación de la Diversidad Entomológica en Iberoamérica (pp. 171-182). Zaragoza, España: Monografías Tercer Milenio.

Reyes-Castillo, P. \& Amat-García, G. (2003). Passalidae (Coleoptera) de Colombia. In G. Onore, P. ReyesCastillo, \& M. Zunino (Eds.), Escarabeidos de Latinoamérica: Estado del conocimiento (pp. 35-50). Zaragoza, España: Monografías Tercer Milenio.

Reyes-Castillo, P. (2004). La tribu Passalini (Coleoptera: Passalidae, Passalinae) en México (Tesis de doctorado). Universidad Autónoma Metropolitana. México, D. F.

Reyes-Castillo, P., Rojas-Gómez, C. V., \& Vázquez, H. (2006). Patrones de distribución de la familia Passalidae (Coleoptera). In J. J. Morrone \& J. LlorenteBousquets (Eds.), Componentes bióticos principales de la Entomofauna Mexicana (pp. 237-270). México, D.F.: Las Prensas de Ciencias, UNAM.

Seeman, O. D., \& Walter, D. E. (1997). A new species of Triplogyniidae (Mesostigmata: Celaenopsoidea) from Australian rainforests. International Journal Acarology, 23, 49-59.
Sinha, R. N., \& Wallace, H. A. H. (1966). Association of Granary Mites and Seed-Bornes Fungi in Stored Grain and in Outdoor and Indoor Habitats. Annals of the Entomological Society of America, 59, 1171-1181.

Schuster, R. O., \& Summers, F. M. (1978). Mites of the family Diarthrophallidae (Acari: Mesostigmata). International Journal of Acarology, 4, 279-385.

Szymkowiak, P., Górski, G., \& Bajerlein, D. (2007). Passive dispersal in arachnids. Biology Letters, 44, 75-101.

Trägärdh, I. (1950). Studies on Celaenopsidae, Diplogyniidae and Schizogyniidae (Acarina). Arkiv For Zoologi, 2(1), 361-451.

Villegas-Guzmán, G. A., Pérez, T. M., \& Reyes-Castillo, P. (2008). Ácaros asociados al coleóptero Passalus cognatus (Coleoptera: Passalidae) de LosTuxtlas, Veracruz, México. Revista de Biología Tropical, 56, 1261-1268.

Villegas-Guzmán, G. A., Reyes-Castillo, P., \& Pérez, T. M. (2011). New species of Klinckowstroemia Baker y Wharton, 1952 (Acari: Klinckowstroemiidae), associated with passalid beetles from Mexico and Panama. Zootaxa, 2747, 19-36.

Villegas-Guzmán, G. A., Francke, O. F., Pérez, T. M., \& Reyes-Castillo, P. (2012). Coadaptación entre los ácaros (Arachnida: Klinckowstroemiidae) y coleópteros Passalidae (Insecta: Coleoptera). Revista de Biología Tropical, 60, 599-609.

Wallace, M. M. H. (1986). Some macrochelid mites (Acari: Macrochelidae) associated with Australian dung beetles (Coleoptera: Scarabaeidae). Acarología, 27, 3-15. 
